Coulomb trajectory effects on electron-impact detachment of negative ions and on mutual neutralization cross sections

This content has been downloaded from IOPscience. Please scroll down to see the full text. 1996 J. Phys. B: At. Mol. Opt. Phys. 296175

(http://iopscience.iop.org/0953-4075/29/24/023)

View the table of contents for this issue, or go to the journal homepage for more

Download details:

IP Address: 140.113.38.11

This content was downloaded on 28/04/2014 at 13:45

Please note that terms and conditions apply. 
J. Phys. B: At. Mol. Opt. Phys. 29 (1996) 6175-6184. Printed in the UK

\title{
Coulomb trajectory effects on electron-impact detachment of negative ions and on mutual neutralization cross sections
}

\author{
J T Lin $\dagger$, T F Jiang $\ddagger$ and C D Lin $\ddagger \S$ \\ $\dagger$ Department of Electrophysics, National Chiao-Tung University, Hsin-Chu, Taiwan 30050, \\ Republic of China \\ $\ddagger$ Institute of Physics, National Chiao-Tung University, Hsin-Chu, Taiwan 30050, Republic of \\ China \\ $\S$ Department of Physics, Kansas State University, Manhattan, KS 66506, USA
}

Received 6 June 1996, in final form 5 August 1996

\begin{abstract}
Cross sections of electron-impact detachment of negative ions and of mutual neutralization between positive and negative ions are calculated using a model based on the semiclassical theory for ion-atom collisions but modified to include Coulomb trajectory effects. It is shown that the method allows us to obtain accurate results for collisions down to low energies. The Coulomb repulsion between the electron and the negative ion accounts for the rapid decrease of detachment cross sections at low energies, and the Coulomb attraction between positive and negative ions accounts for the rapid increase of neutralization cross sections at low velocities.
\end{abstract}

\section{Introduction}

In two recent papers, Andersen et al (1995) and Vejby-Christensen et al (1996) reported electron-impact detachment cross sections of $\mathrm{D}^{-}$and $\mathrm{O}^{-}$ions at energies from threshold to about $30 \mathrm{eV}$ in a merged-beam experiment carried out using the ASTRID Storage Ring. The experimental result shows that the electron detachment cross sections are nearly flat at higher energies but decrease rapidly when the threshold is approached. Compared to the earlier experiments done in the 1970s (Walton et al 1970, 1971, Peart and Dolder 1973), the new experiments were able to measure detachment cross sections near the threshold and refuted the existence of resonances in $\mathrm{D}^{2-}$ claimed in earlier experiments (Walton et al 1970, 1971) and theories (Taylor and Thomas 1971, Thomas 1974). The lack of such resonances has been addressed more recently in the $R$-matrix calculation (Robicheaux et al 1994) and in calculations based on the hyperspherical approach (Yang et al 1996).

The electron-impact detachment of negative ions near threshold has not been extensively examined so far. The threshold law derived using quantum theory by Hart et al (1957) predicted a cross section which increases exponentially with energy but its region of validity is not known. To explain the observed energy dependence of electron-impact detachment cross sections, Andersen and co-workers developed a simple classical reaction theory and they were able to explain the measured cross sections with two fitted parameters. A semiclassical theory based on the tunnelling model (Demkov and Drukarev 1965, Smirnov and Chibisov 1966), has also been used to discuss electron-impact detachment of negative ions. However, the latter fails to account for the experimental results. On the other hand, both theories demonstrated the importance of accounting for the Coulomb repulsion between 
the two negative charged particles in describing electron-impact detachment processes at low collision energies.

While a rigorous treatment of electron-impact detachment of negative ions near threshold requires a full quantum mechanical theory, in this paper we show that a simple semiclassical treatment including the Coulomb repulsion on the electron's trajectory can explain the measured total detachment cross sections over the whole range from near the threshold up to the highest measured energy point at about $30 \mathrm{eV}$. In this approach we treat the incident electron as a classical particle but the active target electron is treated in a quantum mechanical description. We will show that the rapid decrease of detachment cross sections at low energies can be accounted for primarily by the effect of Coulomb repulsion on the classical trajectory of the incident electron. Partial justification for treating the incident electron classically is given later.

We have also applied the semiclassical theory to study total neutralization cross sections between positive and negative ions for centre-of-mass energy down to about $1 \mathrm{eV}$. In this case the Coulomb attraction between the positive and negative ions is to increase the neutralization cross section at lower energies. Results from such calculations for $\mathrm{He}^{+}$on $\mathrm{H}^{-}$and $\mathrm{Li}^{+}$on $\mathrm{D}^{-}$collisions will be shown and compared with the measured results of Peart and Hayton (1994) which covered the centre-of-mass energy from 0.6 to about $300 \mathrm{eV}$.

The basic theoretical model is briefly described in section 2 . The results from the semiclassical calculations are compared with the measurements for electron-impact detachment cross sections of $\mathrm{D}^{-}$and $\mathrm{O}^{-}$in section 3.1 and for neutralization cross sections of $\mathrm{He}^{+}$on $\mathrm{H}^{-}$and $\mathrm{Li}^{+}$on $\mathrm{D}^{-}$in section 3.2. A brief summary concludes the paper. Atomic units are used unless otherwise noted.

\section{Theoretical model}

The starting point of this model is the standard close-coupling theory for treating ion-atom collisions using atomic orbitals as basis functions (Fritsch and Lin 1991). Calculations are carried out within the impact parameter formalism using a one-active-electron approximation for the target negative ion. The time-dependent Schrödinger equation describing the collision on target ion $\mathrm{A}$ by a projectile $\mathrm{B}$, with potentials $U_{A}$ and $U_{B}$, respectively, is

$$
\left[-\frac{1}{2} \nabla_{r}^{2}+U_{A}\left(r_{A}\right)+U_{B}\left(r_{B}\right)-\mathrm{i} \frac{\partial}{\partial t}\right] \Psi=0
$$

where the distances $r_{A}$ and $r_{B}$ are the position vectors of the active electron with respect to the target centre $\mathrm{A}$ and projectile centre $\mathrm{B}$, respectively. The potential $U_{A}$ describing the negative ion is taken to be a short-range Yukawa-type central potential,

$$
U_{A}(r)=-\frac{\mathrm{e}^{-\beta r}}{r} \text {. }
$$

To describe electron-impact detachment on negative ions, we solve equation (1) by expanding the time-dependent wavefunction as

$$
\Psi(b, \vec{r}, t)=\sum_{j=1}^{N_{A}} a_{j}^{A}(b, t) u_{j}^{A}
$$

where the atomic basis functions $u_{j}^{A}$ include the ground state and a set of pseudostates approximately representing the continuum states of the detached electron. Substitution of equation (3) into equation (1) and projecting out to the space of the basis set gives a set of coupled first-order differential equations for the expansion coefficients. The coupled 
equations are solved with a known initial condition and given trajectory to obtain total detachment probability for each impact parameter. In most of the applications in ionatom collisions the trajectory of the incident particle is taken to be a straight line. This approximation is invalid for incident electrons in the low-energy region being considered. The Coulomb repulsion between the incident electron and the negative ion would deflect the electron along a curved trajectory. Instead of rewriting the computer code to include a curved trajectory in the calculation we made the following approximation. For each impact parameter $b$, the Coulomb repulsion would result in a distance of closest approach $r_{c}$ for projectile with incident energy $E$,

$$
r_{c}= \pm \frac{1}{2} D_{0}+\sqrt{\left(\frac{1}{2} D_{0}\right)^{2}+b^{2}}
$$

where $D_{0}=1 / E$ with $E$ being the incident energy, and the positive (negative) sign is for the repulsive (attractive) Coulomb force.

If we assume that detachment occurs only near the distance of closest approach, then the easiest way to account for Coulomb deflection is to interpret the probability for detachment at impact parameter $b$ in a straight-line trajectory calculation to be the detachment probability at the distance of closest approach in a curved trajectory calculation. This substitution has the effect of shifting the transition probabilities to smaller impact parameters when the Coulomb repulsion is considered and thus to reduce the detachment cross sections at low energies.

The above consideration implies that the cross section for neutralization between positive and negative ions will be enhanced if the Coulomb attraction between the ions is included. The deflection is such that the range of impact parameters is increased by the Coulomb attraction even if the range of the distances of closest approach remains the same.

For the neutralization process the electron in the final states is on the projectile. Thus

the time-dependent wavefunction should be expanded in terms of basis functions, $u_{j}^{A}$ and $u_{j^{\prime}}^{B}$, on the two collision centres,

$$
\Psi(b, \vec{r}, t)=\sum_{j=1}^{N_{A}} a_{j}^{A}(b, t) u_{j}^{A} \eta_{j}^{A}+\sum_{j^{\prime}=1}^{N_{B}} a_{j^{\prime}}^{B}(b, t) u_{j^{\prime}}^{B} \eta_{j^{\prime}}^{B}
$$

where $\eta_{j}^{C}$ is the plane-wave electron translational factor (ETF) for atomic states associated with centre $C=\mathrm{A}, \mathrm{B}$.

Calculations for neutralization cross sections in $\left(\mathrm{He}^{+}, \mathrm{Li}^{+}\right)+\mathrm{H}^{-}$collisions have been carried out by Ermolaev (1992) at energies above about $100 \mathrm{eV} \mathrm{amu}^{-1}$ using a straight-line trajectory. We apply the Coulomb correction to the trajectory and extend the calculation to energies below $1 \mathrm{eV}$.

\section{Results and discussion}

\subsection{Detachment cross sections}

We first discuss electron-impact detachment cross sections of $\mathrm{D}^{-}$. The experimental results are shown in figure 1 as open circles. In the theoretical calculations two model potentials were adopted for describing $\mathrm{D}^{-}$. In the first case, we use the Yukawa potential, equation (2), adopted from the work of Ermolaev (1992) where the parameter $\beta=0.8815$. The binding energy obtained from this model potential is -0.02759 au which is to be compared to the experimental value of -0.02756 au. In the close-coupling calculation, besides the ground state, a number of pseudostates are included and they are listed in table 1. The 


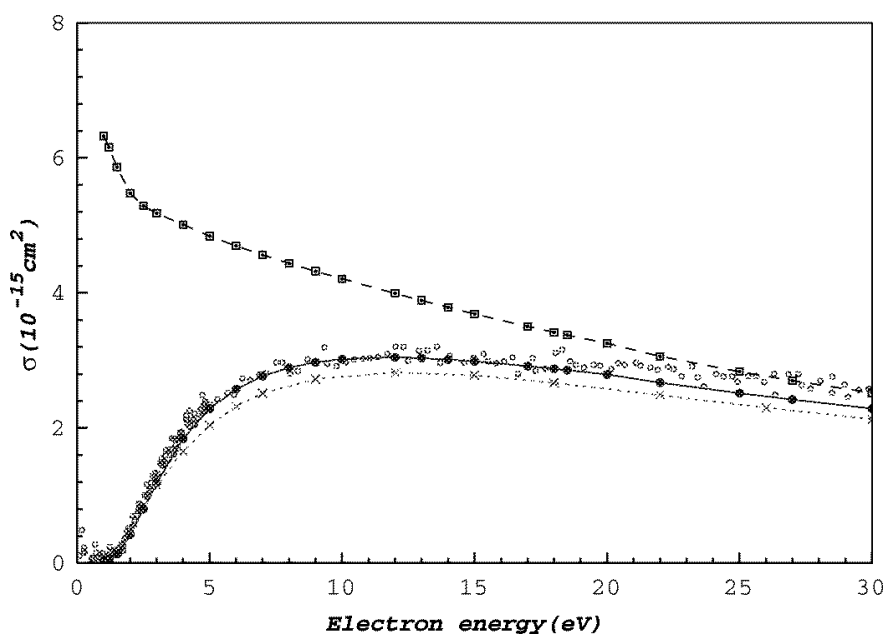

Figure 1. Electron impact detachment cross section of $\mathrm{D}^{-}$as a function of collision energy. Experimental data are from Vejby-Christensen et al (1996). The broken curve is from the present semiclassical calculation assuming straight-line trajectories and the full curve gives the results after including the Coulomb trajectory effect, using a Yukawa model potential for $\mathrm{D}^{-}$. The dotted curve is calculated with a screened model potential for $\mathrm{D}^{-}$including Coulomb trajectory effect.

Table 1. The first two columns give the states and energies of the atomic basis functions in the calculation. The last two columns give the parameters of the Slater orbitals used to diagonalize the model Hamiltonian.

\begin{tabular}{lrll}
\hline$n l$ & $\varepsilon_{n}(\mathrm{au})$ & $n_{j}$ & $\alpha_{j}$ \\
\hline $1 \mathrm{~s}$ & -0.02759 & 0 & 2.5000 \\
$2 \mathrm{~s}$ & 0.00696 & 0 & 1.6666 \\
$3 \mathrm{~s}$ & 0.04334 & 0 & 0.3000 \\
$4 \mathrm{~s}$ & 0.06996 & 1 & 0.3333 \\
$5 \mathrm{~s}$ & 0.19423 & 1 & 1.2500 \\
& & 2 & 0.2500 \\
& & 2 & 0.7500 \\
& & 3 & 0.5000 \\
& & & \\
$2 \mathrm{p}$ & 0.00512 & 1 & 0.6666 \\
$3 \mathrm{p}$ & 0.03103 & 1 & 0.1250 \\
$4 \mathrm{p}$ & 0.17719 & 2 & 1.2500 \\
& & 3 & 0.3333 \\
& & & \\
$3 \mathrm{~d}$ & 0.02534 & 2 & 0.5000 \\
$4 \mathrm{~d}$ & 0.10444 & 2 & 1.2000 \\
$5 \mathrm{~d}$ & 0.35664 & 3 & 0.3333 \\
& & 3 & 1.0000 \\
\hline
\end{tabular}

total detachment cross sections from such a calculation assuming that the electron follows a straight-line trajectory are shown in figure 1 by a broken curve. The results are in reasonable agreement with experiment for energies above $20 \mathrm{eV}$, but at low energies the cross section goes up with decreasing energy in contradiction with the experimental data. By accounting for the Coulomb repulsion on the incident electron's trajectory in the manner discussed 
in the previous section, we obtained new total detachment cross sections. The results are shown as a full curve in figure 1, and are in excellent agreement with experimental data over the whole range of energies shown. The Coulomb trajectory has little effect on the cross sections at higher energies, but it clearly accounts for the decrease of total detachment cross sections at low energies. For easier visualization we did not include results from the classical reaction model calculation which employed two fitting parameters, nor the results from the semiclassical tunnelling model. The detachment cross sections obtained from the latter model are not in good agreement with data. The readers are referred to Vejby-Christensen et al (1996) for details of these two models.

The good agreement between experiment and the above semiclassical calculation for electron-impact detachment cross sections, as shown in figure 1, is indeed astonishing. To make sure that this agreement is not entirely fortuitous we performed another calculation with a somewhat different model potential for describing the $\mathrm{D}^{-}$. We have used a new screened potential $U(r)=-(1+a r) \exp (-2 a r) / r$ where $a=0.6973$ for describing the $\mathrm{D}^{-}$. This potential gives a binding energy of $-0.02779 \mathrm{au}$, somewhat deeper than the experimental value of -0.02756 au and the value -0.02759 au obtained from the Yukawa potential earlier. The resulting cross sections after correcting for the Coulomb trajectory effect are shown as a dotted curve in figure 1. The overall energy dependence is still very good even though the actual values are 5-10\% lower. Note that this new model potential gives a more tightly bound electron for $\mathrm{D}^{-}$and thus the resulting detachment cross section is somewhat lower. We emphasize that the general energy dependence is very insensitive to the model potential used. The simpler Yukawa potential gives better binding energy for $\mathrm{D}^{-}$and better detachment cross sections at higher energies. It will be used for the rest of this paper.

To illustrate the effect of Coulomb repulsion on the calculated detachment probabilities, we show in figure 2 the comparison of the weighted detachment probabilities versus impact parameters at two energies, one at $15 \mathrm{eV}$ and the other at $2 \mathrm{eV}$. The effect of the curved trajectory clearly is much more significant at the lower energies.

In figure 3 we show the calculated detachment probabilities versus impact parameters at a number of incident energies (Coulomb repulsion is included). The probabilities drop smoothly from near zero impact parameters over a range of the order of about $15 \mathrm{au}$. The detachment probability reaches a value of about of 0.5 or 0.6 at the higher energies, but drops to less than 0.1 at the lower energy point of $2 \mathrm{eV}$. This is to be compared to the classical reaction model of Vejby-Christensen et al (1996) where a uniform probability of about 0.2 was ascribed for detachment probability for all energies.

Vejby-Christensen et al (1996) also reported electron-detachment cross sections of $\mathrm{O}^{-}$ ions from the threshold to $30 \mathrm{eV}$. They also have performed calculations based on the classical reaction model and the tunnelling model. The classical reaction model obtained with fitting parameters was able to describe the measured detachment cross sections above $5 \mathrm{eV}$, but gave results in disagreement with data at lower energies. The tunnelling model fails to reproduce the data completely. We have also studied electron-detachment cross sections of $\mathrm{O}^{-}$ions. In this case the outermost electron occupies an open-shell $2 \mathrm{p}$ orbital. To approximate such a system as one active electron in a model central potential is more precarious. Furthermore, we have found it very difficult to obtain a model potential for $\mathrm{O}^{-}$ ions where the active electron has only one bound $\mathrm{p}$ orbital without introducing additional bound orbitals for other angular momentum states. We are thus forced to adopt a simpler model where the outermost electron in $\mathrm{O}^{-}$is treated as a $1 \mathrm{~s}$ electron in a Yukawa potential of the form $-\frac{Z}{r} \exp (-\alpha r)$. By choosing $Z=3.2$ and $\alpha=3.3335$, we were able to obtain one single bound state with energy -0.05402 au which is to be compared to the experimental 

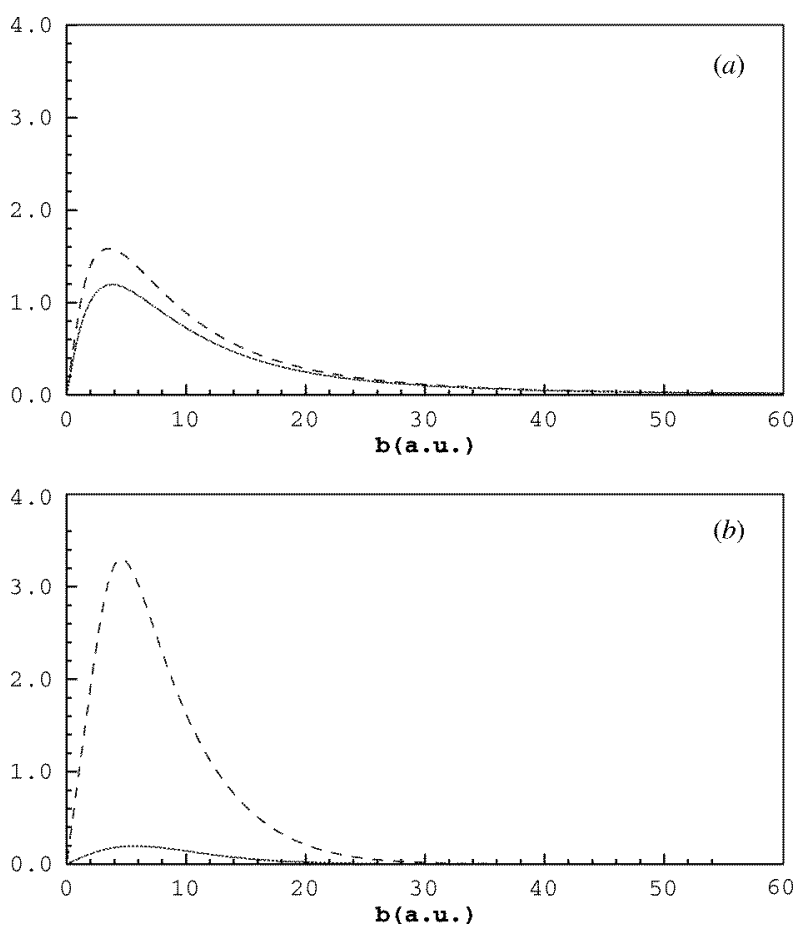

Figure 2. Weighted detachment probabilities versus impact parameters, with (full curve) and without (broken curve) Coulomb repulsion for electron collisions with $\mathrm{D}^{-}$at (a) $15 \mathrm{eV},(b) 2 \mathrm{eV}$.

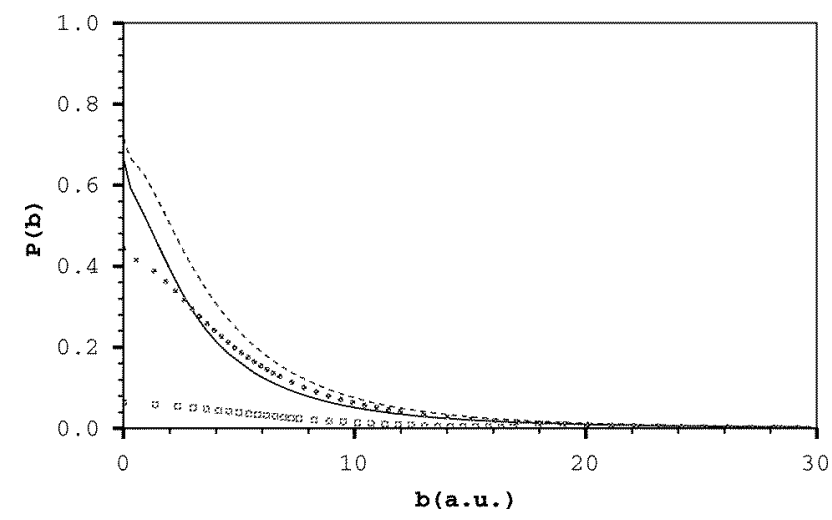

Figure 3. Detachment probabilities versus impact parameters for electron- $\mathrm{D}^{-}$collisions with Coulomb repulsion at different energies. Full cuvre, $30 \mathrm{eV}$; broken curve $10 \mathrm{eV}$; full circles, $5 \mathrm{eV}$; open squares, $2 \mathrm{eV}$.

energy of -0.0537 au. By performing close-coupling calculations and accounting for the Coulomb trajectory effect, the resulting detachment cross sections are shown as a full curve in figure 4. While the overall energy dependence is similar to the experimental data, the actual values are about $10-15 \%$ higher over the whole energy range. In view of the limited information on the model potential for this system and the fact that we treated the outermost electron as a 1s electron, the calculated total detachment cross section can be considered to 


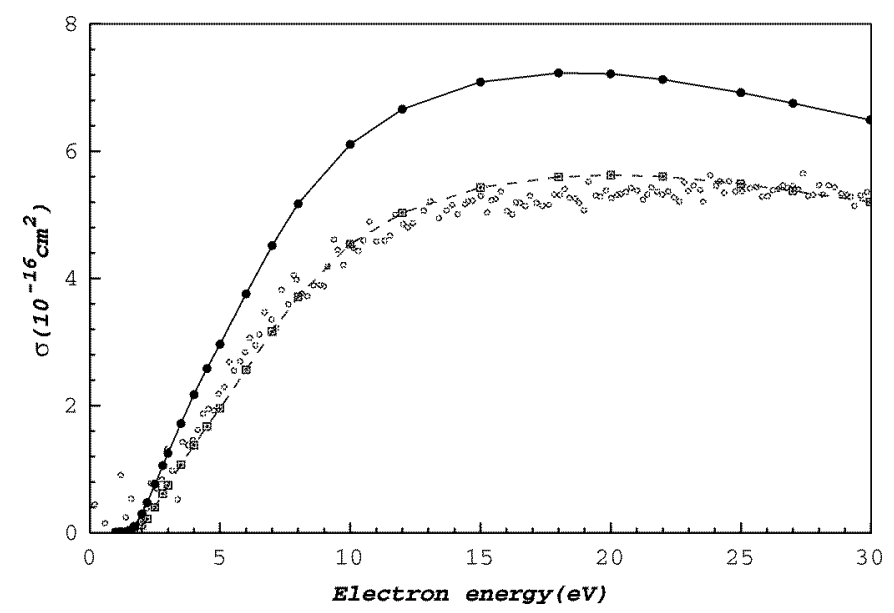

Figure 4. Detachment cross sections for electron- $\mathrm{O}^{-}$collisions from 1 to $30 \mathrm{eV}$. Experimental data are from Vejby-Christensen et al (1996). Theoretical calculations: full curve, from a model potential which gives a binding energy of -0.05402 au for $\mathrm{O}^{-}$which is close to the experimental value of $-0.0538 \mathrm{au}$; broken curve, from a less realistic model potential which gives a binding energy of $-0.06337 \mathrm{au}$. The latter model was chosen to fit the detachment cross sections at higher energies.

be satisfactory. Further improvement may require treating the $\mathrm{O}^{-}$ion as a many-electron system.

To illustrate that the energy dependence of detachment cross sections is indeed determined mostly by the Coulomb trajectory effect, we artificially change the fitting parameters of the above model potential until the actual calculated detachment cross sections are in good agreement with experimental data at higher energies. The final parameters thus obtained are $Z=3.3$ and $\alpha=3.410$, and the corresponding binding energy for this model potential is -0.06337 au which is $18 \%$ deeper than the actual experimental value. With the model potential parameters thus fixed we then carried out the calculations at all the energies and the resulting cross sections, after the correction due to the Coulomb repulsion, are shown as broken curves in figure 4 . Notice that in this case the resulting cross sections are in good agreement with experiment over the entire energy range, proving that the Coulomb trajectory effect included in our approach indeed can account for the energy dependence. The cross section of $\mathrm{O}^{-}$is about one-fifth of that of $\mathrm{D}^{-}$due to its higher binding energy and its ground state is more localized than $\mathrm{D}^{-}$.

From figures 1 and 4 we can conclude that the present semiclassical model with correction due to the Coulomb trajectory does give accurate description of the energy dependence of the electron-impact detachment cross sections of negative ions, even down to the threshold region. Thus if the semiclassical calculation is able to obtain correct detachment cross sections at the higher energies, the resulting cross sections using the present model appear to be valid for the whole energy region down to near the threshold.

One can obviously raise a number of objections to this model, including treating the incident electron classically. This procedure probably can be partially justified in that the range of impact parameters involved in the collision is quite large and the de Broglie wavelength is small compared to the range of interaction even at relatively low velocities. The small detachment energy of atomic negative ions probably also implies that the failure of the semiclassical model will occur at smaller energies-at energies below $1 \mathrm{eV}$. The 
experimental data available so far do not explore this energy region adequately. While a full quantum formulation of the electron-impact detachment of negative ions near the threshold is certainly desirable, the long-range nature of atomic negative ions also implies that a large number of partial waves is needed in a quantal calculation which further justifies the use of the semiclassical theory.

\subsection{Neutralization cross section}

The result in the previous section illustrates that the Coulomb repulsion between the electron and the negative ion is responsible for the rapid decrease of detachment cross sections at low energies. One can immediately expect that the Coulomb attraction between positive and negative charged particles at low energies would result in an enhancement of cross sections. The most important process in low-energy collisions between positive and negative charged particles is the mutual neutralization process. In this subsection we examine the effect of Coulomb attraction on mutual neutralization cross sections.

We consider the recent measurements by Peart and Hayton (1994) where mutual neutralization cross sections for $\mathrm{He}^{+}+\mathrm{H}^{-}$and $\mathrm{Li}^{+}+\mathrm{D}^{-}$collisions have been measured for centre-of-mass energies from 0.6 to $258 \mathrm{eV}$ and from 0.7 to $316 \mathrm{eV}$, respectively. Cross sections for these two systems have been calculated using the standard semiclassical model by Ermolaev (1992) for energies above about $100 \mathrm{eV} \mathrm{amu}^{-1}$ using atomic basis functions, and by Méndez et al (1990) for energies above $30 \mathrm{eV} \mathrm{amu}^{-1}$ using molecular basis functions.

The experimental mutual detachment cross sections for $\mathrm{He}^{+}+\mathrm{H}^{-}$are shown in figure 5(a). We performed a semiclassical calculation using the same basis set and model potentials (see table 2 of Ermolaev 1992) as employed by Ermolaev at centre-of-mass energy $0.2 \mathrm{keV}$. The mutual neutralization cross section calculated at this energy agrees with his result. Since we have numerical difficulties in extending the calculations to lower collision energies, we decided to take the calculated total mutual neutralization probabilities versus impact parameters as constant, i.e. we assume that the probabilities remain the same at all the lower energies in a straight-line trajectory calculation. From the relation between the distances of closest approach and impact parameters for each energy we calculated the mutual neutralization cross sections at lower energies down to about $1 \mathrm{eV}$. The results are shown in the full curve in figure 5(a). It is clear that the increase of mutual neutralization cross sections is nicely reproduced considering that the extrapolation has been applied from collision energy at $200 \mathrm{eV}$ down to $1 \mathrm{eV}$ without any new scattering calculation. We see a discrepancy occuring near about $10 \mathrm{eV}$. This is probably due to the assumption we made that the probabilities remain the same for all the energies calculated. This assumption is not entirely correct since at lower energies the neutralization process can populate different final states. From table 3 of Ermolaev (1992), we see that the final state distributions do change as the collision energy is varied.

We have also applied the same procedure to $\mathrm{Li}^{+}+\mathrm{D}^{-}$collisions and the results are shown in figure $5(b)$. The actual close-coupling calculation was performed at the centre-ofmass energy of $375 \mathrm{eV}$. At this energy there is a 9\% discrepancy from experimental data and thus the extrapolated total mutual neutralization cross sections over the indicated energies are somewhat lower. If we apply the same correction due to the Coulomb curved trajectory to obtain total neutralization cross sections down to about $0.5 \mathrm{eV}$, the results are shown as a full curve in figure $5(b)$. We also notice some non-eligible discrepancy at energies around $10 \mathrm{eV}$. The discrepancy is likely due to the change of the population of different final states as the collision energy is reduced. However, the effect of attractive Coulomb force indeed is to raise the neutralization cross sections at low energies. 

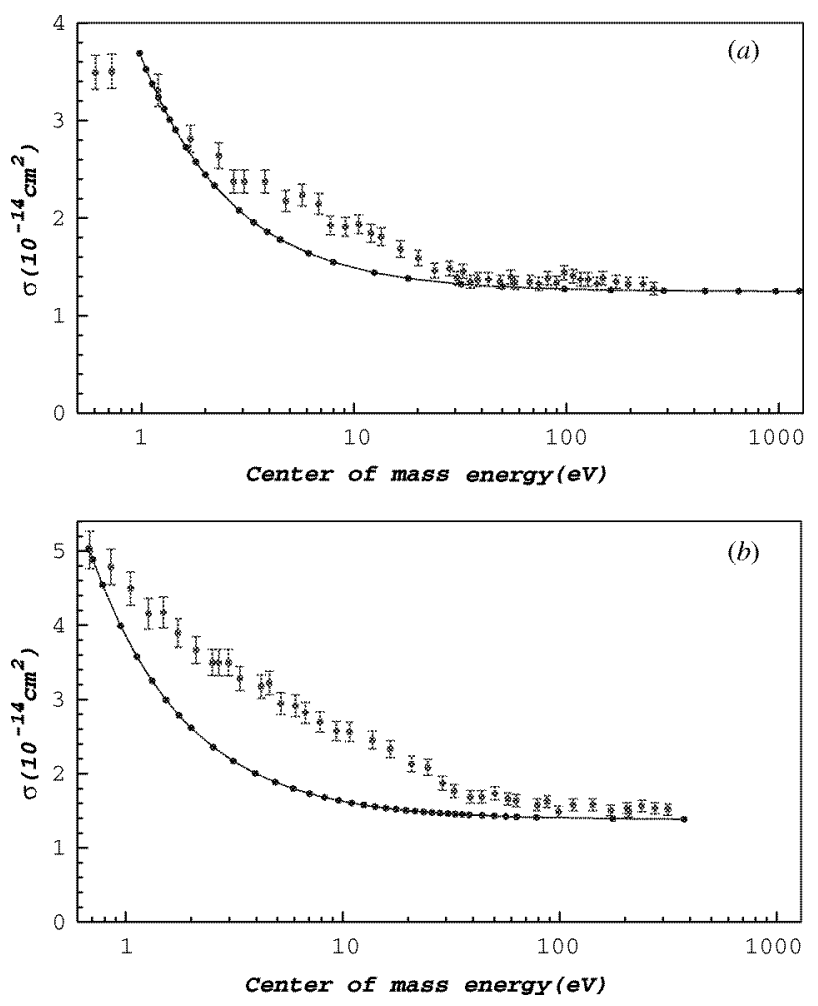

Figure 5. Mutual neutralization cross sections for $(a) \mathrm{He}^{+}$on $\mathrm{H}^{-}$. Experimental data from Peart and Hayton (1994). The full curve is from the present calculation carried out at $E=0.200 \mathrm{keV}$ folded with effect from the Coulomb trajectory. $(b) \mathrm{Li}^{+}$on $\mathrm{D}^{-}$. Experimental data from Peart and Hayton (1994). The full curve is from the present calculation carried out at $E=0.375 \mathrm{keV}$ folded with effect from the Coulomb trajectory.

\section{Summary}

We have illustrated that the total detachment cross sections for electrons colliding with negative ions and total mutual neutralization cross sections between positive and negative ions can be described using a semiclassical theory down to about $1 \mathrm{eV}$ centre-of-mass energies if the Coulomb trajectory effect is included. In this model, the transition probabilities stay constant or nearly constant versus distances of closest approach and the enhancement or the reduction of cross sections is attributed entirely to the Coulomb trajectory effect. The good agreement between this model calculation and the experimental data indicates that this is the major factor for determining the variation of total cross sections at low energies. This model is expected to be valid for the dominant transitions or processes occurring at large internuclear separations and offers an easy estimate of electron-impact detachment or mutual neutralization cross sections at low energies.

Note added in proof. After this paper was submitted for publication, Ostrovsky and Taulbjerg (1996) published a new model for electron-impact detachment of negative ions by combining the quantum tunnelling model of Smirnov and Chibisov (1966) and the classical above-barrier transition model for field ionization. They obtained good agreement with experimental data at low energies but not at higher energies and the results are more sensitive to the model potentials used for describing the $\mathrm{D}^{-}$ions than what we have found. 


\section{Acknowledgments}

This work is supported in part by the National Research Council of Taiwan under the contract number NSC85-2811-M009-004. TFJ and CDL are also partly supported by a US-Taiwan cooperative research program.

\section{References}

Andersen L H, Mathur D, Schmidt H T and Vejby-Christensen L 1995 Phys. Rev. Lett. 74892

Demkov Y N and Drukarev 1965 Sov. Phys.-JETP 20614

Ermolaev A M 1992 J. Phys. B: At. Mol. Opt. Phys. 253133

Fritsch W and Lin C D 1991 Phys. Rep. 2021

Hart R W, Gray E P and Guier W H 1957 Phys. Rev. 1081512

Méndez L, Copper I P, Dickson A M, Mó O and Riera A 1990 J. Phys. B: At. Mol. Opt. Phys. 232797

Ostrovsky V N and Taulbjerg K 1996 J. Phys. B: At. Mol. Opt. Phys. 292573

Peart B and Dolder K 1973 J. Phys. B: At. Mol. Phys. 61497

Peart B and Hayton D A 1994 J. Phys. B: At. Mol. Opt. Phys. 272551

Robicheaux F, Wood R P and Greene C H 1994 Phys. Rev. A 491866

Smirnov B M and Chibisov M I 1966 Sov. Phys.-JETP 22585

Taylor S H and Thomas L D 1971 Phys. Rev. Lett. 281091

Thomas L D 1974 J. Phys. B: At. Mol. Phys. 7 L97

Vejby-Christensen L, Kella D, Mathur D, Pedersen H B, Schmidt H T and Andersen L H 1996 Phys. Rev. A 53 2371

Walton D S, Peart B and Dolder K 1970 J. Phys. B: At. Mol. Phys. 3 L148

1971 J. Phys. B: At. Mol. Phys. 41343

Yang X, Bao C G and Lin C D 1996 Phys. Rev. Lett. 743069 